\title{
Médiévales
}

Langues, Textes, Histoire

70 | printemps 2016

Lieux d'hygiène et lieux d'aisance en terre d'Islam (VII $\mathrm{e}_{-}$ $\mathrm{XV}^{\mathrm{e}}$ siècle)

\section{Soulagement au château : les lieux d'aisance dans les fortifications du Proche-Orient médiéval}

Relief in the Castle: The Latrines Rooms in the Near Eastern Medieval

Fortifications

Cyril Yovitchitch

\section{OpenEdition \\ Journals}

Édition électronique

URL : http://journals.openedition.org/medievales/7831

DOI : 10.4000/medievales.7837

ISSN : $1777-5892$

Éditeur

Presses universitaires de Vincennes

Édition imprimée

Date de publication : 23 juin 2016

Pagination : 149-169

ISSN : 0751-2708

Référence électronique

Cyril Yovitchitch, «Soulagement au château : les lieux d'aisance dans les fortifications du Proche-

Orient médiéval », Médiévales [En ligne], 70 I printemps 2016, mis en ligne le 15 juin 2018, consulté le

09 mai 2019. URL : http://journals.openedition.org/medievales/7831 ; DOI : 10.4000/medievales.7831 
Cyril Yovitchitch

\section{Soulagement au château : les lieux d'aisance dans les fortifications du Proche-Orient médiéval}

Noyés dans la masse architecturale des fortifications, rarement étudiés, au mieux signalés lors des descriptions archéologiques, les lieux d'aisance restent avant tout des espaces sombres et peu attrayants. Pourtant, l'étude systématique de leur localisation et de leur disposition permet de tirer un certain nombre d'enseignements sur les programmes architecturaux militaires et leurs concepteurs.

\section{Les lieux d'aisance dans le programme défensif et résidentiel}

Dans les forteresses du Proche-Orient, les latrines se rencontrent dans divers espaces tels que les tours, les courtines, les portes d'entrée principales, où elles sont adaptées à la nature du programme défensif ou résidentiel.

\section{Dans les courtines et tours d'enceinte}

Les circuits défensifs ne sont pas toujours régulièrement pourvus de dispositifs d'aisance bâtis, et leur logique d'implantation n'est pas souvent évidente à comprendre, car les latrines sont tantôt construites dans les tours, tantôt dans les courtines. Sur l'enceinte orientale de la ville du Caire, érigée sous Saladin à la fin du XII ${ }^{\mathrm{e}}$ siècle, on observe, entre les deux grosses tours d'angle Burdj al-Zafar et Burdj al-Mạ̣rūq, plusieurs tourelles dont le rezde-chaussée est doté de latrines. Afin de ne pas créer de point faible, ces dernières sont aménagées dans l'épaisseur du mur arrière des tours ; elles étaient accessibles depuis un couloir d'entrée ouvert dans leur gorge en face duquel débouchait parfois un escalier conduisant au chemin de ronde (fig. 1). Dans ce cas, les latrines étaient situées à l'articulation entre deux 

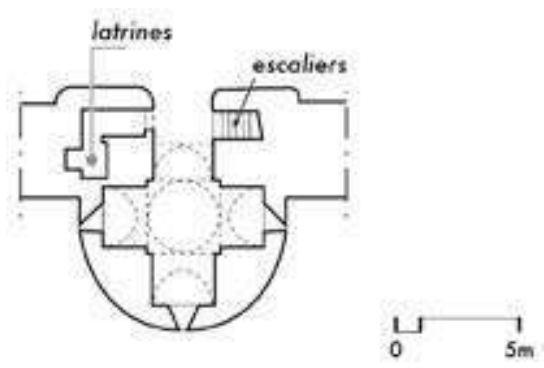

Fig. 1. Le Caire. Enceinte orientale. Première tour au sud de Bāb al-Djadīd (Dessin C. Yovitchitch 2015, d'après K. A. C. Creswell 1952)

zones de défense (les tours et le système de circulation qui reliait les deux niveaux de combat). Au sud de Burdj al-Mahrūq, le programme défensif change et, comme dans la citadelle, des gaines de circulation apparaissent dans les courtines à partir desquelles on accédait aux latrines. Leur position dans les gaines supérieures de l'enceinte pourrait s'expliquer par le souci des bâtisseurs d'éviter de fragiliser les parties basses de murs qui étaient la cible des équipes de sapeurs.

On ne retrouve pas ces caractéristiques dans les fortifications croisées contemporaines, où les tours semblent plus rarement dotées de latrines. Les tourelles semi-circulaires des enceintes extérieures des grandes forteresses hospitalières du Crac des Chevaliers ou du Marqab en sont dépourvues à l'exception de la tourelle $\mathrm{n}^{\circ} 10$ de l'enceinte extérieure de la première qui en présente trois dans le massif adossé au nord ${ }^{1}$. Le nombre et la distribution des lieux d'aisance sur les différents niveaux de la forteresse devaient être jugés suffisants, notamment en raison de l'existence d'une vaste tour de latrines (voir infra).

Dans les lieux de défense éloignés des espaces à caractère résidentiel, on a souvent pris soin d'aménager des espaces de soulagement. C'est le cas de l'enceinte basse orientale du château de 'Adjlūn, qui renferme des latrines dans une archère accolée à la tour d'angle sud-est, mais aussi de la grande salle de défense de l'enceinte basse du château de Kérak où des latrines occupent l'extrémité de la salle. Enfin, il arrive que les lieux d'aisance soient disposés le long des couloirs comme à Qal'at Nadjim, où

1. J. Mesqui, Forteresses médiévales au Proche-Orient. Quatre châteaux des hospitaliers au nord du comté de Tripoli. Le Crac des Chevaliers (Qal'at al-Hoșn), Margat (Qal'at al-Marqab), Castrum Rubeum (Qal'at Yahmūr), Coliath (al-Qulay'āt), chap. 2, La troisième enceinte, 2003, p. 18 (version électronique http://castellorient.fr/0-Accueil/ indexfran.htm). 
le grand couloir nord, qui traverse d'ouest en est le château, est pourvu de trois latrines ${ }^{2}$.

Le souci de disposer des lieux d'aisance dans ou à proximité des grosses tours défensives est une des caractéristiques de la fortification ayyoubide et mamelouke. On retrouve ce principe au Crac des Chevaliers, où l'extension quadrangulaire de la tour sud-ouest (construite sous Baybars [1223-1277]) est traversée par un couloir d'accès sur lequel s'articule l'espace des latrines (fig. 2) ${ }^{3}$.

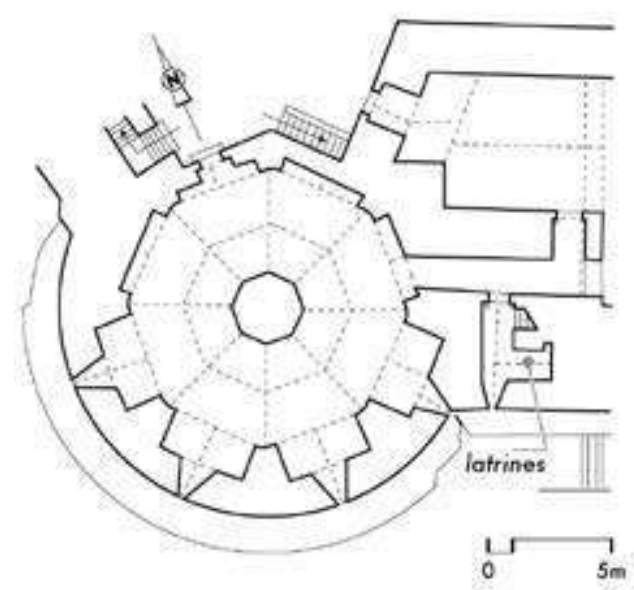

Fig. 2. Crac des Chevaliers, tour sud-ouest

(Dessin C. Yovitchitch 2015, d'après P. Deschamps 1934)

\section{Dans les portes}

$\mathrm{Au}$ Proche-Orient, les entrées des forteresses ou des enceintes urbaines sont majoritairement ouvertes dans des tours-portes associées à une tour flanquante, le passage se faisant au travers de la base de la tourporte selon un schéma plus ou moins complexe ${ }^{4}$. Les latrines, quand elles existent, ne sont alors pas directement accessibles depuis le passage, mais

2. J. A. MARTínez LóPEZ, « Del castro romano al castillo árabe : Qal'at Naŷm, una fortificación en la frontera del Éufrates », dans A. GonZÁLEZ BLANCO, G. MATILLA SÉQUIER éd., Romanización y Cristianismo en la Siria Mesopotámica, Antigüedad y Cristianismo, 15 (1998), p. 437-464 ; C. Yovitchitch, « Qal'at Najm, forteresse-palais des bords de l'Euphrate », Chronos, 23 (2011), p. 105-140.

3. J. MESQUI, Forteresses médiévales..., chap. 2, La troisième enceinte, p. 13.

4. Sur les entrées des forteresses islamiques médiévales proches-orientales, voir C. Yovitchitch, Forteresses du Proche-Orient. L'architecture militaire des Ayyoubides, Paris, 2011, p. 217-259. 
le plus souvent situées à l'étage de la porte et dans la tour flanquante qui complète le programme défensif. Dans ce cas, c'est tout le système d'entrée qui offrait les conditions nécessaires à une station prolongée sans être fragilisé par une urgence naturelle irréfrénable. Au Caire, deux portes de l'enceinte orientale adoptent ce schéma. Les latrines sont placées dans le mur arrière de la tour flanquante, selon un plan déjà observé pour les tourelles de l'enceinte. Alors que le couloir d'entrée des latrines de Bāb al-Djadīd démarre au niveau du vestibule d'entrée de la tour, celui de Bāb al-Barqiyya se greffe au fond de la niche de l'archère nord (fig. 3).

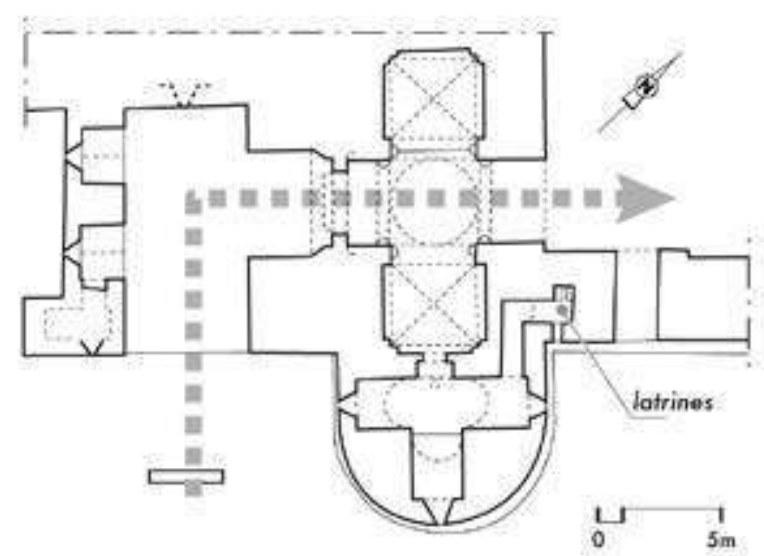

Fig. 3. Le Caire. Enceinte orientale, Bāb al-Djadīd 2 (Bāb al-Barqiyya)

(Dessin C. Yovitchitch 2015, d'après N. Warner 1999, S. Pradines 2002 et observations personnelles)

\section{Dans les tours maitresses}

Au tournant du XIII ${ }^{\mathrm{e}}$ siècle, on assiste dans la fortification islamique proche-orientale à une augmentation considérable de la taille et du volume des tours construites par les princes musulmans et leurs émirs. Ces ouvrages n'avaient pas pour seule fonction la défense, ils servaient aussi de cadre de vie aux officiers ou au prince lors de son séjour et parfois même de prison pour des personnes de haut rang ${ }^{5}$. Ces grosses tours disposaient donc des aménagements nécessaires à la résidence et à l'incarcération, parmi lesquels figuraient les latrines. Ainsi, dans les années 1200, le sultan ayyoubide al-Malik al-'Ādil, fraîchement installé à la tête de la dynastie, entreprit de renforcer les défenses des citadelles de Damas et de Bosra afin d'asseoir son pouvoir. À cette occasion, des tours aux dimensions gigantesques sortirent de terre, dépassant les vingt à trente mètres de côté,

5. Ibid., p. 202. 
dans lesquelles s'imbriquaient étroitement les fonctions de défense et de résidence, voire de représentation ${ }^{6}$. À Bosra, les deux immenses tours qui emboîtent les angles nord-ouest et nord-est de la citadelle ne sont pourvues de latrines que dans les espaces résidentiels. Dans la première, seul l'étage en possède ; elles sont associées à une petite pièce située en retrait par rapport au reste des salles logeables. Dans la seconde, l'étage renfermait au sud-est un large espace de vie. Les latrines furent implantées en dehors, à proximité de l'entrée, afin de les isoler et de les rendre accessibles depuis l'espace de vie commun.

Cette façon de rejeter les latrines à l'extérieur des volumes habitables est illustrée au château de Qal 'at Nadjm sur l'Euphrate, dans ses deux grandes tours orientales ${ }^{7}$. Près de la tour nord-est, les latrines sont situées au bout du couloir qui offrait un exutoire de proximité tout en éloignant les odeurs du lieu de vie. À quelques mètres de l'entrée de la mosquée, elles pouvaient en outre servir à ceux qui désiraient faire leurs ablutions avant la prière. Près de la tour sud-est, le dispositif est analogue : les latrines étaient accessibles depuis le vestibule d'entrée de la tour, face à l'escalier qui conduisait à la galerie de défense à l'étage. À Baalbek, les latrines de la grosse tour sud de la citadelle sont accessibles depuis l'escalier qui monte à l'étage, là aussi, délibérément rejetées hors des lieux de vie et de réception ${ }^{8}$. À la citadelle du Caire, Creswell identifie quatre latrines à l'étage de la grosse tour Burdj al-Șufa éclairées par une petite ouverture située en hauteur (fig. 4) ${ }^{9}$. Alors que la partie projetée en avant de la muraille n'est occupée que par des archères à niche, le restant de la tour, situé à l'arrière, du côté du corps de place, présente une distribution double : un premier vaste espace intérieur articulé sur un plan cruciforme à $i w a \bar{n} n$-s sur l'un desquels vient se raccorder au sud un petit couloir desservant trois latrines, et un second espace à vocation résidentielle ou carcérale (?), doté de plusieurs petites salles dont des latrines. Burdj Karkyalān, une autre grosse tour de la citadelle du Caire, possède des latrines rejetées dans son angle arrière gauche afin de bien les isoler des espaces de vie, tandis que l'autre angle arrière est dévolu à la circulation verticale ${ }^{10}$. Dans cette distribution des espaces internes, deux blocs se dessinent : l'un, situé en avant, pour les activités défensives et

6. C. Yovitchitch, Forteresses du Proche-Orient..., p. 177-213 ; ID., «Ornements et représentation du pouvoir dans la fortification ayyoubide », dans Guerre et paix dans le Proche-Orient médiéval, $X^{e}-X V^{e}$ siècles. Histoire, archéologie et anthropologie, Colloque international tenu au Caire, 15-18 décembre 2011, à paraître.

7. C. Yovitchitch, «Qal'at Najm... », p. 115.

8. T. WIEGAND, Baalbek. Ergebnisse der Ausgrabungen und Untersuchungen in den Jahren 1898 bis 1905, 3 vol., Berlin/Leipzig, 1925.

9. K. A. C. CRESWEll, The Muslim Architecture of Egypt, vol. 2, Ayyûbids and Early Bahrite Mameluks A.D. 1171-1326, [1959], New-York, 1978, p. 9-10.

10. K. A. C. CReswell, Ayyûbids..., p. 11. 


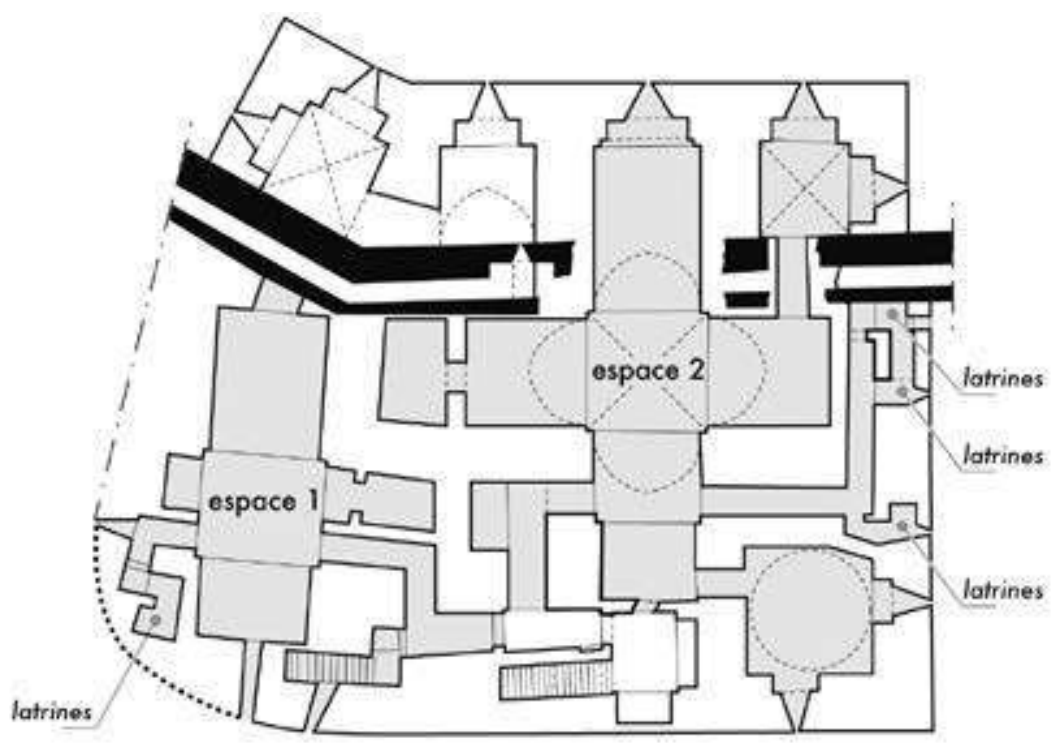

Fig. 4. Burdj al-Șufa

(Dessin C. Yovitchitch 2015, d'après K. A. C. Creswell 1952)

résidentielles, l'autre, situé en arrière, où sont concentrées les activités polluantes et de circulation.

Au château de 'Adjlūn, en 1214, fut construite une grande tour qui combinait, sur plusieurs niveaux, des aménagements défensifs et résidentiels ${ }^{11}$. Ces derniers sont caractérisés par l'usage de larges baies quadrangulaires et par la création d'espaces à caractère privé. Ainsi, au rezde-chaussée, des latrines sont associées à une chambre isolée en bout de salle, ce qui renforce l'hypothèse du caractère privé de ces lieux d'aisance tenus à l'écart de la soldatesque.

Parfois, c'est l'existence de latrines qui offre une indication sur l'usage résidentiel de certaines tours dont le caractère défensif semble prépondérant. Ainsi, les grandes tours barlongues du front sud de la citadelle de Damas, construites au début $\mathrm{du} \mathrm{XIII}^{\mathrm{e}}$ siècle, sont-elles dotées à chaque étage de latrines, au point que l'on peut se demander si ces pièces ne servaient pas de dortoir aux soldats de la garnison. C'est aussi le cas de cette grande salle à archères mamelouke du Crac des Chevaliers, identifiable à un dortoir

11. C. N. Johns, « Medieval 'Ajlun : I. The Castle (Qal'at ar-Rabad)», Quarterly of the Department of Antiquities of Palestine, 1 (1931), p. 21-33 ; C. YovitchiтcH, « The Tower of Aybak in 'Ajlûn Castle : An Exemple of the Spread of an Architectural Concept in Early 13th Century Ayyubid Fortification », dans H. KENNEDY éd., Muslim Military Architecture in Greater Syria. From the Coming of Islam to the Ottoman Period, Leyde, 2006, p. 225-242 ; ID., Forteresses du Proche-Orient..., p. 188. 
associé à un appartement pour un officier situé dans son prolongement. Les latrines qui lui étaient adossées, rejetées à l'extérieur, n'étaient accessibles que depuis la rampe d'accès ${ }^{12}$.

Dans les grandes forteresses croisées, les grosses tours sont elles aussi dotées de latrines. Sur le front sud du Crac des Chevaliers, s'élevaient, au XIII ${ }^{\mathrm{e}}$ siècle, trois imposantes tours réservées aux dignitaires de la forteresse. Construites sur plusieurs niveaux, elles accueillaient des espaces aux fonctions spécifiques : grande pièce, dortoir, salle d'apparat, systématiquement pourvus de dispositif de soulagement ${ }^{13}$. Au Marqab, une grosse tour circulaire adossée à une vaste salle formait le logis des chevaliers ; les latrines étaient situées dans le mur ouest de la tour, accessibles depuis une niche d'archère ${ }^{14}$.

\section{Éléments pour une typologie des latrines}

Les petits réduits qui accueillent les latrines se rangent en deux principales catégories : ceux qui adoptent un plan axial (en «I») et ceux qui dessinent des angles simples (en « L »), multiples (en « $\mathrm{S} »)$ ou en «baïonnette », afin de soustraire à la vue l'occupant des lieux (fig. 5). Les premiers se rencontrent plutôt dans la fortification croisée, les seconds étant communs aux deux aires culturelles. On peut également classer les latrines en fonction du mode d'évacuation des déjections. Ainsi on distingue trois grands types de latrines : à fosse, en encorbellement et à conduit biais ${ }^{15}$.

\section{Les types d'évacuation}

Dans les latrines à fosse, les matières étaient recueillies dans de grandes fosses bâties qui étaient régulièrement vidées. L'avantage de ce dispositif était d'éviter que les fèces ne s'accumulent au pied des ouvrages comme cela était le cas pour les deux autres types d'évacuation. L'inconvénient majeur résidait dans la remontée des effluves que l'on tentait de juguler par l'usage d'un système d'aération (qui aspirait la colonne d'air

12. J. MESQui, Forteresses médiévales..., chap. 2, La troisième enceinte, p. 6.

13. P. DesCHAMPS, Les Châteaux des Croisés en Terre Sainte, 1, Le Crac des Chevaliers, Paris, 1934, p. 189-192, 205-213.

14. P. DesChAmps, Les Châteaux des Croisés en Terre sainte, 3, La Défense du comté de Tripoli et de la principauté d'Antioche, Paris, 1973, p. 280-281 ; J. MESQUI, Margat, al-Marqab, 2003-2004, publication électronique, http://castellorient.fr/0-Accueil/indexfran. htm.

15. J. MESQui et N. FAUCHÈrE, «L'hygiène dans les châteaux forts au Moyen Âge », La Vie de château. Les Cahiers de Commarque, Le Bugue, 1992, p. 45-74. 


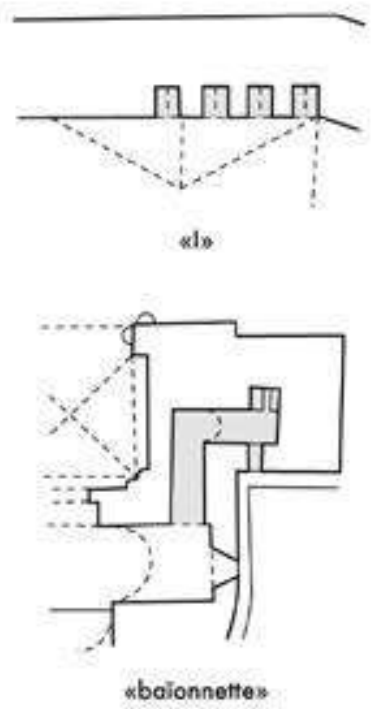

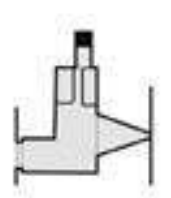

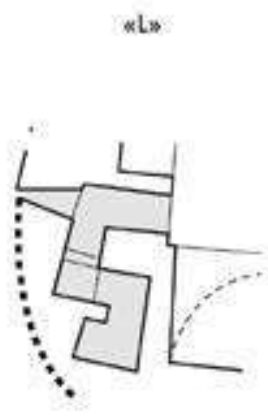

(4)

Fig. 5. Les principaux types de latrines

(Dessin C. Yovitchitch 2015)

impur vers le bas) et à l'aide d'un couvercle bouchant l'orifice ${ }^{16}$. Ce type de latrines existe, mais n'est pas très fréquent dans les fortifications du ProcheOrient médiéval. On peut citer celles du Crac des Chevaliers mamelouk situées dans la rampe d'accès (voir supra ${ }^{17}$.

Tandis que les latrines en encorbellement s'imposent en Occident au $\mathrm{XIII}^{\mathrm{e}}$ et au XIV ${ }^{\mathrm{e}}$ siècles, ce n'est pas un type d'évacuation des plus fréquents dans la fortification islamique. Creswell pense pouvoir en identifier une sur l'enceinte fatimide du Caire, entre Bāb al-Nașr et la première tour à l'ouest, mais cette interprétation porte à discussion ${ }^{18}$. L'édicule en question s'apparente à une bretèche, comme souvent pour les latrines en encorbellement. Les corbeaux des cinq consoles sont ornés, mais ceci n'est pas discriminant en soi. Ce qui pose problème, c'est qu'il ne s'agit pas d'une structure à simple, mais à quadruple orifice (mâchicoulis), et qu'aucune séparation ne semble avoir existé entre eux. De plus l'orientation de l'édicule, tourné exactement en direction de la qibla, semble incompatible avec la pratique de l'Islam (voir infra). Peut-être faut-il la comprendre

16. Ibid., p. 56.

17. Les latrines à fosse sont connues dans les habitations domestiques, comme à Qașr al-Hayr al-Sharqī, où plusieurs maisons en possèdent dans les pièces dédiées aux ablutions, voir D. GENEQUAND, Les Établissements des élites omeyyades en Palmyrène et au ProcheOrient, Beyrouth, 2012, p. 122.

18. K. A. C. CRESwEll, vol. 1, Ikhîds and Fâtimids A.D. 939-1171, [1952], New-York, 1978 , p. 185. 
comme étant un complément du programme ornemental déployé sur la porte nord.

Pour les périodes ayyoubide et mamelouke, les latrines en encorbellement ne sont guère plus fréquentes. Peut-être peut-on en identifier une à l'angle sud-ouest de la tour nord-ouest de la citadelle de Bosra. Elle se présentait sous la forme d'un conduit en chicane qui débouchait en avant de la courtine primitive. Au château de Shayzar, on peut observer deux latrines en encorbellement, superposées, qui desservaient le premier et le deuxième étage de la grande tour mamelouke érigée au sud de l'arête rocheuse. Celle du premier étage est effondrée, mais les quelques éléments qui subsistent suffisent à comprendre qu'elle était construite sur le même modèle que celle de l'étage supérieur qui est bien conservée. L'encorbellement se présente sous la forme d'une boîte de « mâchicoulis » reposant sur un alignement de trois consoles. Le canal d'évacuation est taillé dans les corbeaux médians. On s'interroge en revanche sur la datation de celles de la tour en partie reconstruite sous les Mamelouks au château de Șaydā, cette dernière n'ayant pas fait l'objet d'une étude approfondie ${ }^{19}$.

Les latrines à conduit biais constituent le principal type d'évacuation employé dans les forteresses islamiques et croisées du Proche-Orient. Le conduit peut sortir en hauteur, mais le plus souvent il descend à la verticale dans l'épaisseur du mur et débouche à sa base. Les conduits desservent fréquemment plusieurs latrines superposées, comme à 'Adjlūn, Bosra et Damas. Au Crac des Chevaliers, le groupe de huit latrines qui occupe un pan nord de la première enceinte s'évacue par une large ouverture au pied du mur. Quant aux douze conduits de la tour des latrines, située juste à côté, ils s'évacuent dans les trois mâchicoulis sur arc qui ouvrent sur sa face nord.

\section{Les systèmes de rinçage}

Les latrines en encorbellement ou à conduit biais ne nécessitaient pas de système de rinçage complexe, mais l'adhérence de certaines sécrétions devait fortement inciter à verser de l'eau pour nettoyer les conduits. Ceci ne devait cependant pas suffire à masquer les odeurs ni à empêcher les nombreuses mouches de tournoyer dans la pièce ${ }^{20}$. On peut s'interroger sur les odeurs régnant aux abords de la tour des latrines du Crac des Chevaliers.

19. M. PianA, « Die Kreuzfahrerstadt Sidon (Sagette, Saidâ) », dans ID., Burgen und Stätdte der Kreuzzugszeit, Petersberg, 2008, p. 367-383. L'encorbellement est orienté nordsud ; aussi, s'il appartient à la phase de restauration mamelouke, il offre un contre-exemple à l'idée que l'occupant des latrines ne devait pas être tourné vers la Mecque (vide infra).

20. P. Mitchell, J. Huntley, E. STERN, «Bioarchaeological Analysis of the 13th Century Latrines of the Crusader Hospital of St. John at Acre, Israel », dans V. MALLIAMiLANES éd., The Military Orders. 3. History and Heritage, Aldershot, 2008, p. 213-223 (p. 216). 
En effet, même s'il faut garder à l'esprit que la délicatesse de notre nez n'était pas nécessairement celle d'un soldat du Moyen Âge, la chaleur intense de l'été au Proche-Orient contribuait, à n'en pas douter, à neutraliser assez rapidement les effluves émanant des matières fécales tout en les rendant plus aisées à ramasser. En revanche, durant les mois plus humides, et surtout dans les latrines fermées, il devait en être tout autrement, aussi faisait-on usage de chaux pour éteindre les odeurs et accélérer le processus de dégradation ${ }^{21}$.

C'est peut-être pour profiter des pluies hivernales que certaines latrines n'étaient pas couvertes afin de permettre à l'eau d'inonder les conduites. On rencontre un exemple de ce type dans un escalier qui relie le premier étage au couronnement de l'une des grosses tours ayyoubides de la citadelle de Bosra (tour 5). En général, dans les édifices qui possèdent des latrines sur plusieurs niveaux, celles-ci occupent une position identique afin d'utiliser le même conduit d'évacuation. À Damas, les lieux d'aisance des grandes tours du front sud sont situées dans un angle du mur arrière, usant d'une même colonne d'évacuation depuis les galeries de défense jusqu'au rez-de-chaussée. D'autres sites présentent des systèmes un peu plus sophistiqués rendus nécessaires par la position interne des latrines qui ne pouvaient pas s'évacuer rapidement dehors. Ainsi à Qal'at Nadjm les latrines du grand couloir nord sont-elles connectées à une canalisation qui, traversant le château d'est en ouest, devait rassembler les eaux de pluie, celles des citernes et du hammām afin de les rincer. Un système analogue de canalisations qui récoltaient les eaux de pluie pour les diriger vers les latrines se trouve au Crac des Chevaliers dans celles de la première enceinte croisée. Au château de Șubayba, les lieux d'aisance mamelouks de la tour de Bīlīk étaient nettoyés grâce à un réservoir (alimenté par une citerne située juste en dessous) dans lequel on pouvait puiser de l'eau pour chasser les matières vers l'extérieur du mur. L'eau contenue dans ce réservoir, placé à côté des latrines du premier étage, pouvait être également puisée depuis le deuxième étage au travers d'une large conduite verticale ${ }^{22}$.

\section{Privatives ou collectives, avec ou sans dispositifs de fermeture}

Dans les latrines médiévales islamiques, le plan en chicane ou en «L» semble avoir offert une intimité suffisante à l'occupant, et rendu inutile l'usage d'une fermeture dans la majorité des cas. De ce fait, on observe peu de dispositifs de fermeture à l'entrée des couloirs d'accès. La plupart

21. Voir P. Mitchell, J. HunTLEy, E. STERN, « Bioarchaeological Analysis... », p. 213223.

22. M. HARTAL, The al-Subayba (Nimrod) Fortress : Towers 11 and 9, IAA Reports, 11, Jérusalem, 2001, p. 36-38. 
du temps, il faut se contenter d'indices indirects pour déceler l'existence d'un système de fermeture, comme la présence d'un renfoncement dans la maçonnerie, destiné à effacer la porte dans le mur. Lorsque les murs sont dotés de feuillures, il est rare qu'elles soient couplées à des crapaudines, comme dans celles du premier niveau de la tour mamelouke du château de Șubayba ${ }^{23}$.

Les ordres militaires hospitalier et templier avaient pour habitude de construire dans leurs forteresses des tours dédiées aux lieux d'aisance. Il en subsiste quelques exemples au Proche-Orient. Au Crac des Chevaliers, un pôle d'hygiène était concentré dans le secteur septentrional du noyau primitif du château. Au rez-de-chaussée, il occupait la portion nord du mur arrière de l'enceinte, et se présentait sous la forme d'une succession de douze latrines (un groupe de quatre et un groupe de huit) accessibles par un étroit couloir aménagé dans l'épaisseur du mur (fig. 6). Plus tard, c'est une véritable tour qui fut construite dans ce secteur offrant une douzaine de lieux d'aisance supplémentaires aux occupants du niveau supérieur du château. Précédés par une vaste salle, ils se déversaient par un conduit vertical dans trois grands mâchicoulis sur arc (fig. 7) ${ }^{24}$. Une autre série de quatre latrines occupe le flanc droit de la tour, près du chemin d'accès à la poterne. Son entrée est située en dehors de la tour, ce qui pourrait indiquer qu'elle était à destination de ceux qui œuvraient au niveau des lices. Ce pôle d'hygiène était complété par d'autres lieux d'aisance construits au niveau de la seconde enceinte, dans la tourelle $n^{\circ} 10$ située juste en face de la tour des latrines.

On retrouve un programme similaire, bien que moins ambitieux, au château du Marqab, où deux saillants rectangulaires possédaient un bloc de latrines en terrasse. Le premier, à l'ouest de la chapelle, doté de quatre sièges sur deux niveaux ${ }^{25}$; le second, sur l'enceinte orientale ${ }^{26}$. À Saint-Jean d'Acre, la tour des latrines érigée dans le quartier des hospitaliers totalisait trente-cinq sièges. Les conduites, rincées par les eaux de pluie provenant des terrasses, rejoignaient au sous-sol une fosse inclinée qui collectait les eaux usées de plusieurs secteurs avant de se déverser dans un égout qui

23. Ibid.

24. J. MESQUI, Forteresses médiévales..., chap. 3, Le château haut : première et seconde enceinte, p. 14-16. Voir également l'étude de la tour des latrines du Crac par Reinhard Schmitt, et celle des systèmes de nettoyage par Werner Meyer et Maria-Letizia Boscardin dans T. BILler et al., Der Crac des Chevaliers. Die Baugeschichte einer Ordensburg der Kreuzfahrerzeit, Regensburg, 2006, respectivement p. 130-134, et p. 305-343.

25. J. MeSQUI, Margat, al-Marqab, publication électronique, http://castellorient.fr/0Accueil/indexfran.htm, 2003-2004, chap. 2, Le château, p. 10-11 ; B. MAJOR, « Medieval "light construction buildings" on top of the vaulted halls of al-Marqab Citadel (Syria) ", Castelos das orden militares, Lisbonne, 2014, p. 172.

26. B. MAJOR, « Medieval "light construction buildings"... », p. 164-181. 


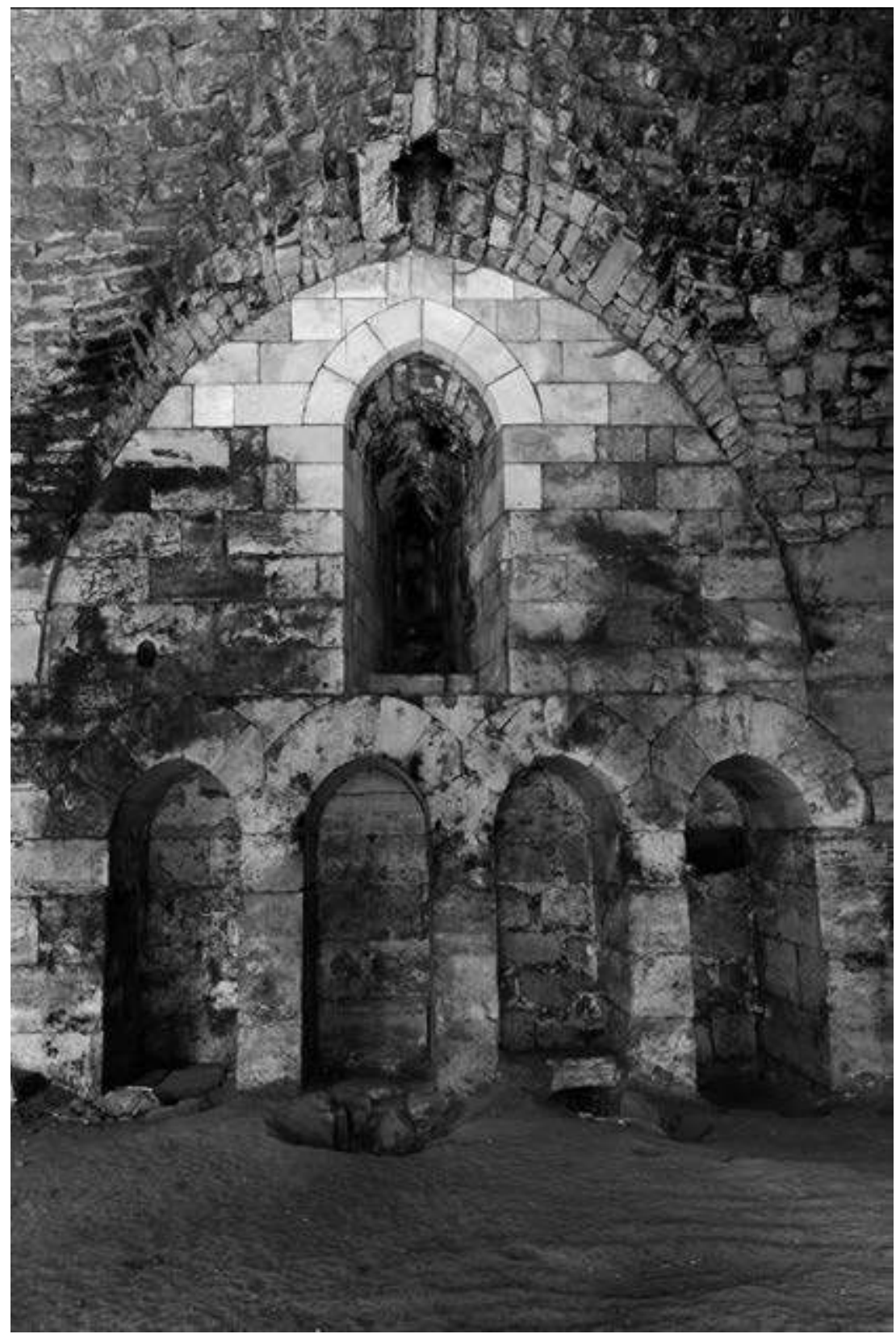

Fig. 6. Crac des Chevaliers.

Bloc de latrines de la première enceinte du château croisé

(Photographie C. Yovitchitch) 


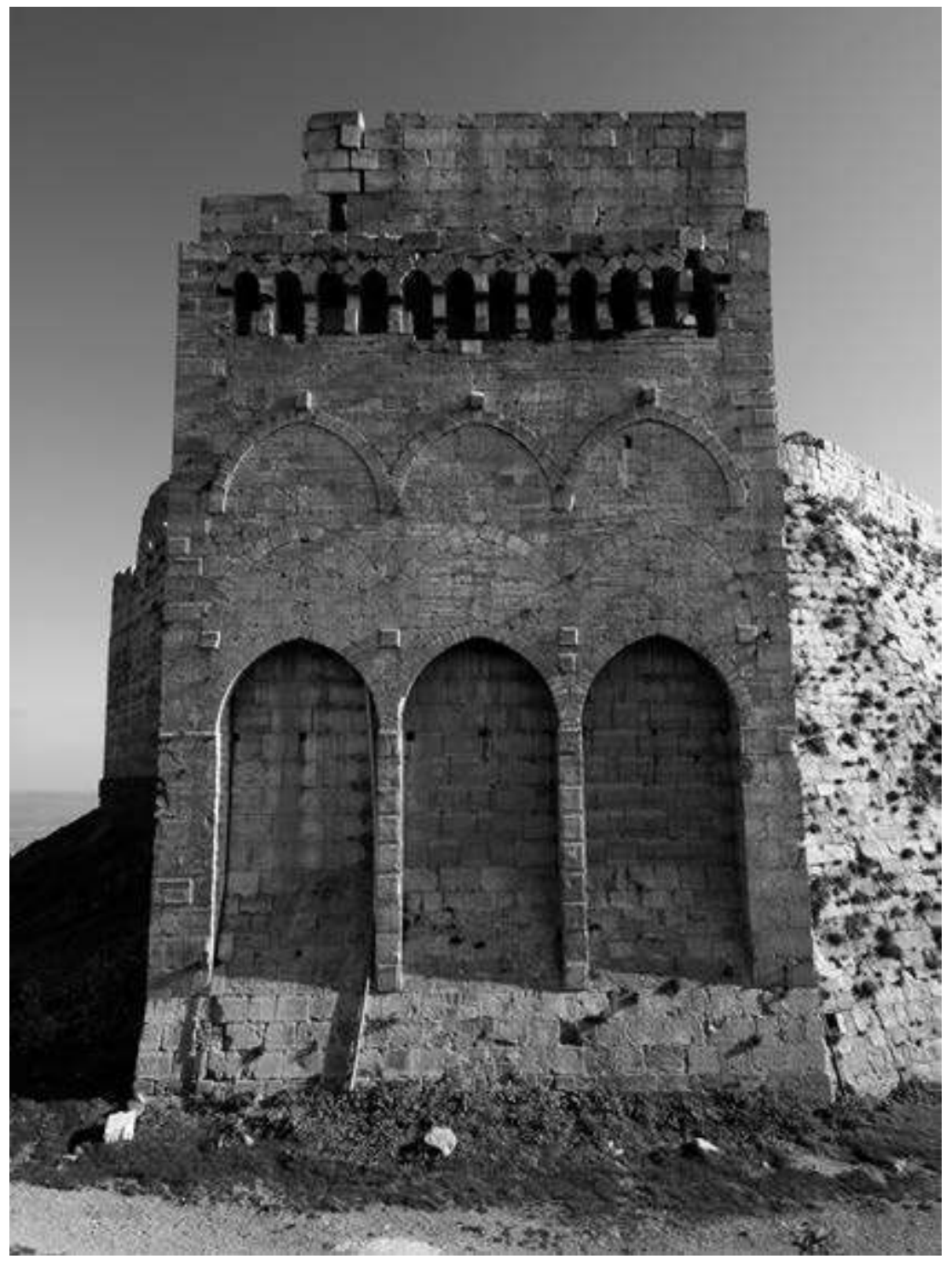

Fig. 7. Crac des Chevaliers.

La tour des latrines du château croisé

(Photographie C. Yovitchitch) 
traversait la ville et se jetait dans la mer ${ }^{27}$. L'enceinte externe du château templier de Șâfītà conserve les restes d'un saillant qui présente les vestiges des trémies d'évacuation d'une douzaine de latrines au niveau du glacis ${ }^{28}$. À Kérak, une salle en partie enfouie au cœur du château croisé possède plusieurs latrines alignées (fig. 8) qui ressemblent à celles de la première enceinte du Crac des Chevaliers.

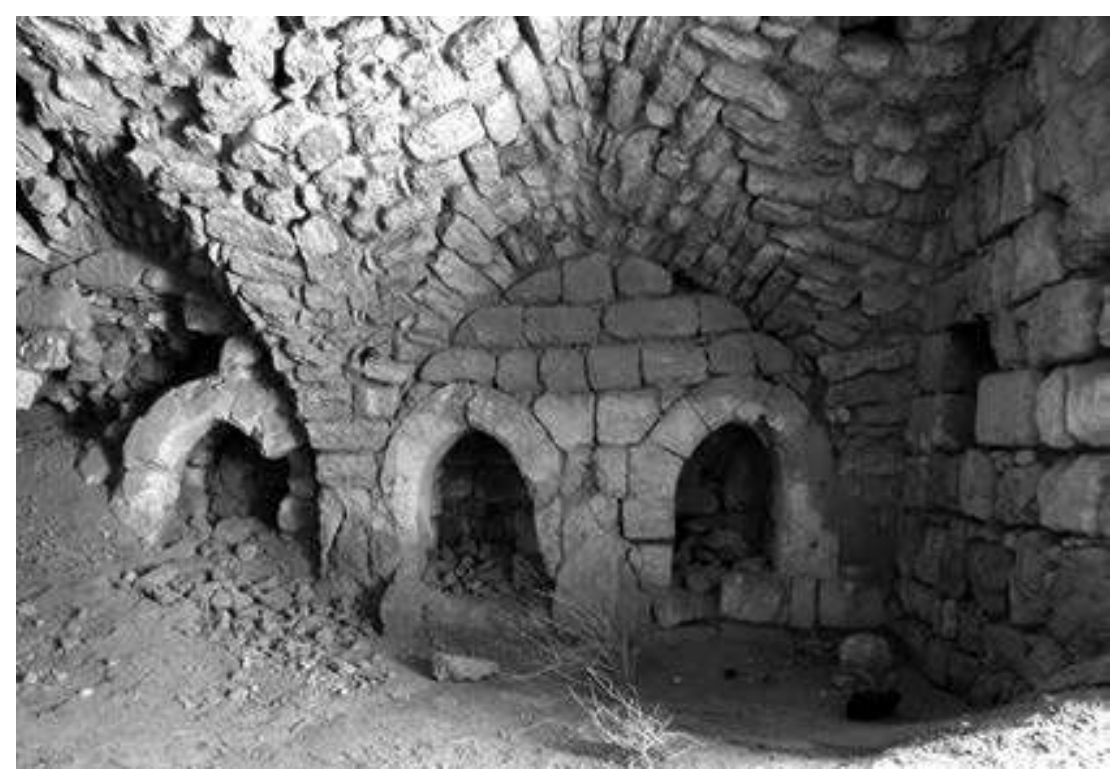

Fig. 8. Château de Kérak. Latrines « collectives » du château croisé

(Photographie C. Yovitchitch)

\section{Dis-moi comment tu te soulages, je te dirai qui tu es}

\section{Accroupi ou assis?}

Certains aménagements relevés par l'étude typologique pourraient être mis en relation avec des interdits religieux. La tradition islamique prescrit plusieurs règles de conduite au croyant ${ }^{29}$. Il lui est notamment demandé de ne jamais se tourner en direction de la Mecque pour uriner ou déféquer. Même si, à l'origine, cette interdiction concerne des pratiques

27. D. JACOBY, « Die Kreuzfahrerstadt Akko », dans M. PiANA éd., Burgen und Stätdte der Kreuzzugszeit..., p. 242-251.

28. M.PIANA, «Die Templerburg Chastel Blanc (Burg as-Sâfîtâ) », dans ID., Burgen und Stätdte der Kreuzzugszeit..., p. 297.

29. Voir l'article de Y. BENHIMA dans ce même volume. 
à l'air libre, force est de constater que, plusieurs siècles plus tard, elle était appliquée à la construction en dur, car la disposition des latrines des forteresses médiévales proche-orientales islamiques suit en général cette règle.

Les latrines islamiques médiévales sont désignées par les locutions : bayt raha, bayt al-khalā', bayt mirḥ̂ăd, mais aussi par celles de kursī raha, kurs̄̄ khalā' ou kurs̄̄ mirhạạ. Si l'on comprend aisément la signification des locutions formées avec le terme bayt, c'est-à-dire de la pièce réservée à l'usage des ablutions et de la toilette, le mot kursī est plus difficile à mettre en relation avec la réalité archéologique et les usages. Les latrines islamiques en contexte fortifié se présentent sous la même forme : deux blocs parallèles séparés d'une vingtaine de centimètres (fig. 9) avec, parfois, un élargissement à l'extrémité (fig. 10). Ce dispositif répond en tout point à la sunna qui recommande d'uriner accroupi afin d'éviter d'être souillé par des projections. Peut-on envisager que certaines latrines aient pu être surmontées d'un siège, comme le suggère le terme kursī ? La question reste ouverte. Néanmoins, il existe des latrines à Qal'at al-Șubayba, Baalbek (fig. 11) ou Șalkhad qui présentent une forme particulière : deux pierres parallèles forment un podium de plus de $50 \mathrm{~cm}$ de haut auquel on accédait par une marche ${ }^{30}$. L'emploi de kursī dans les textes serait peut-être plutôt à relier à cette surélévation.

À l'opposé, les latrines des forteresses croisées possédaient un siège comme il était d'usage en Occident. Ce dernier comportait un trou circulaire ou semi-circulaire taillé dans une banquette en pierre. Alors que très souvent les sièges ont été cassés ou démontés, plusieurs exemples de ce type sont visibles à Saranda Kolones à Chypre, où trois des piliers centraux du château abritent deux latrines disposées dos-à-dos (fig. 12) ${ }^{31}$. Parfois, le siège était fait d'une planche en bois percée, comme en témoignent les rainures horizontales taillées dans les murs latéraux des latrines de la « tour des latrines » du Crac des Chevaliers.

\section{Dans l'intimité des latrines}

Les fouilles des latrines médiévales apportent des renseignements sur les pratiques liées à l'hygiène intime, comme sur les habitudes alimentaires ou sur l'environnement naturel et immédiat. Elles révèlent aussi le piètre état sanitaire des populations qui fréquentaient ces lieux. Ainsi l'étude de celles du château croisé de Saranda Kolones a-t-elle montré une importante

30. M. HARTAL, The al-Subayba ..., p. 36-38 et 46-47; T. WIEGAND, Baalbek..., p. 74, fig. 74 et 76.

31. A. H. S. Megaw, «The Arts in Cyprus : Military Architecture », in K. M. SETton, éd., A History of the Crusades, vol. 4, The Art and Architecture of the Crusader States, Madison (Wisc.), 1977, p. 196-207. 


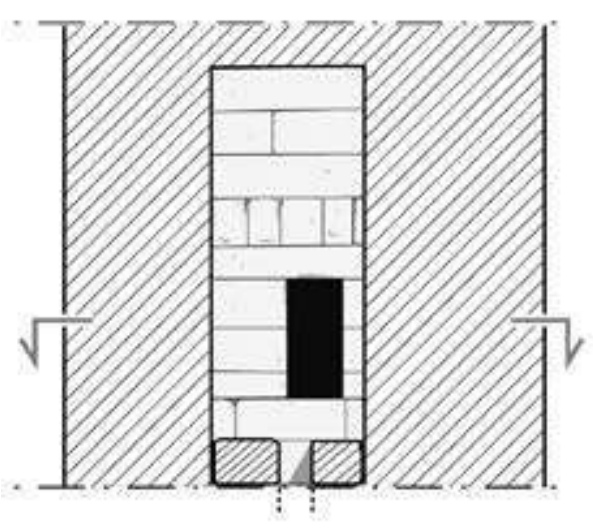

coupe A

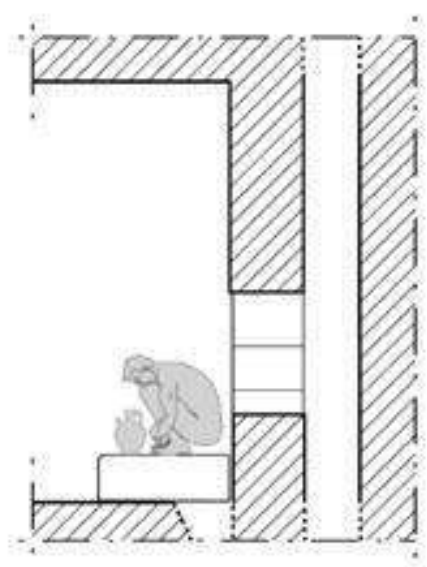

coupe B

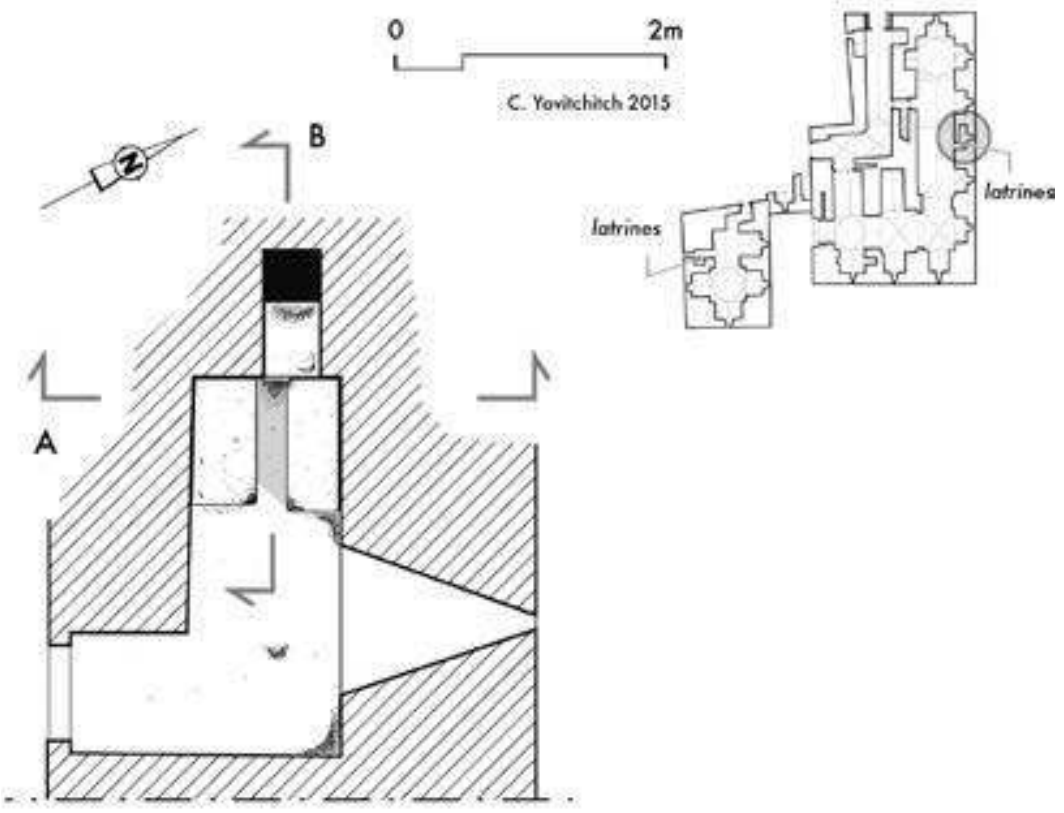

Fig. 9. Citadelle de Bosra.

Latrines en « $\mathrm{L}$ » de la tour d'entrée sud-est

(Dessin C. Yovitchitch 2015) 


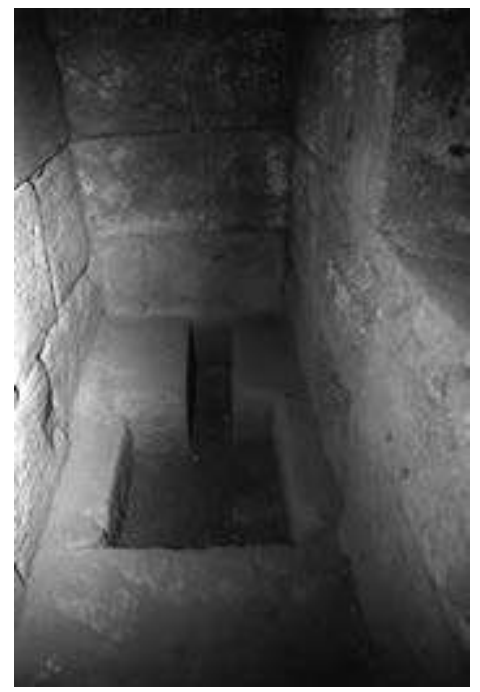

Fig. 10. Citadelle de Bosra, tour 10. Latrines de la salle à archères de l'étage (Photographie C. Yovitchitch)

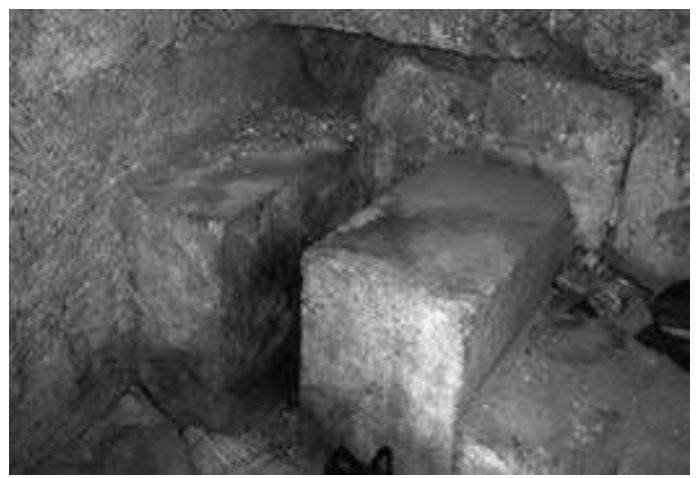

Fig. 11. Citadelle de Baalbek. Latrines mameloukes de l'escalier d'accès à l'étage (Photographie C. Yovitchitch)

parasitose liée à un manque d'hygiène ${ }^{32}$. Elle a révélé par ailleurs la présence de tiges d'herbes et de céréales, de la paille, mise en relation avec l'hygiène intime $^{33}$. La paille pouvant engendrer de fortes démangeaisons, voire des blessures, même chez quelqu'un à la main preste, il est peu probable qu'elle ait été utilisée pour s'essuyer, contrairement à l'étoupe ou à d'autres

32. E. Anastasiou, P. Mitchell, « Human intestinal Parasites from a Latrine in the 12th Century Frankish Castle of Saranda Kolones in Cyprus », International Journal of Paleopathology, 3 (2013), p. 218-223 (p. 222).

33. P. Mitchell, J. Huntley, E. STERN, « Bioarchaeological analysis... », p. 215. 


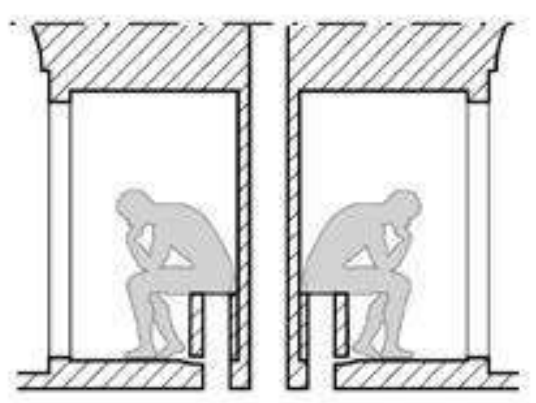

égout

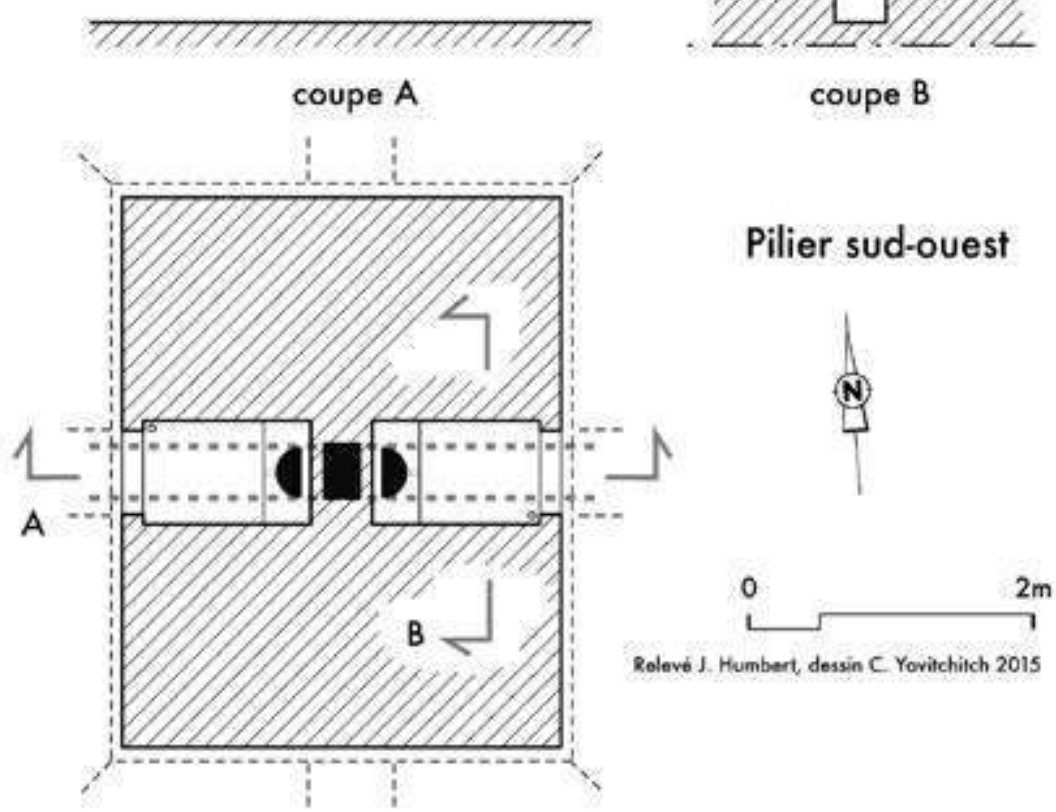

Fig. 12. Saranda Kolones. Latrines adossées du pilier nord-ouest de la salle centrale (Relevé J. Humbert, dessin C. Yovitchitch 2015)

cotonnades. En revanche, elle pourrait avoir servi pour le récurage des conduits, ce qui expliquerait qu'elle se retrouve mélangée aux fèces.

Soulagement dans un cadre raffiné

Si tous ces lieux d'aisance comptent parmi les endroits dans lesquels on s'attarde a priori le moins, il en est un qui laisse le visiteur quelque peu perplexe. Il s'agit des latrines de la grosse tour mamelouke qui se dresse 
sur le front sud du château de Shayzar ${ }^{34}$. Cet espace est couvert par une voûte formant un trilobe qui se termine dans un cul-de-four reposant sur un rang de muqarnas (fig. 13). L'éclairage était fourni par un orifice circulaire d'une vingtaine de centimètres de diamètre percé à mi-hauteur. Ces latrines étaient très certainement réservées à l'usage des dignitaires, tout comme celle de l'étage de la tour mamelouke construite au sud du front oriental du Crac des Chevaliers, qui offrait un cadre délicat, grâce à la voussure ornée en forme de conque de l'une des deux archères qui l'encadraient.

Ce déploiement de raffinement dans un endroit aussi trivial que le lieu d'aisance n'est pas si surprenant quand on fait le rapprochement avec les objets d'une grande finesse qui agrémentaient les latrines de certaines riches demeures bagdadiennes du XII ${ }^{\mathrm{e}}$ siècle. C'est du moins ce que laisse entendre la relation par Ibn al-Athīr du pillage en 570/1174 du palais du grand émir Qutb al-Dīn Qaymāz : « On y vit un raffinement sans pareil. Par exemple, dans son cabinet d'aisance (bayt al-țhāra) se trouvait une chaîne en or [pendant] du plafond jusqu'à arriver en face de l'ouverture des latrines ( $\underline{k h} a l \bar{a})$. Au bout [de cette chaîne] [se trouvait] une grosse boule en or, tout ajourée, remplie de musc et d'ambre, afin que [celui qui] se tenait [sur les latrines] puisse la sentir. Les hommes luttèrent férocement [pour la prendre] et elle fut coupée ${ }^{35}$.»

À l'issue de cette première approche, les lieux d'aisance étudiés apparaissent, par leur disposition et leur composition, comme des marqueurs culturels. Certains répondent aux recommandations de la tradition islamique : l'orientation du corps implique celle de l'espace dédié ; la position du corps induit un support adapté et, enfin, l'impératif de la toilette intime nécessite la présence d'eau. À l'inverse, dans les latrines appartenant à la sphère croisée, l'orientation ne tenait compte que des contraintes liées à la construction des ouvrages et aux programmes architecturaux. Quant au besoin d'intimité, il était relégué au second plan derrière la volonté de rationaliser les espaces de vie et de combats (en particulier dans les grandes forteresses construites

34. M. VAN BERCHEM et E. FATIO, Voyage en Syrie, Le Caire, 1914-1915, vol. 1, p. 177187 ; C. TonghinI, Shayzar 1 : The Fortification of the Citadel, Leyde, 2011, p. 192-193.

35. Je tiens à remercier vivement Vanessa Van Renterghem pour m'avoir signalé et traduit ce passage tiré du Kâmil d'Ibn al-Athîr : IBN AL-ATHîR, al-Kâmil fí l-Ta'rîh, Beyrouth, 1998, vol. 10, p. 71. La subtilité de cet usage oriental du parfum, en particulier du musc, destiné à masquer les effluves prégnantes qui se dégageaient des lieux d'aisance, et qui était aussi prescrit pour la toilette intime, trouve un écho deux siècles plus tard, de l'autre côté de la Méditerranée, à la cour royale de France où, dans un compte de 1396, il est question de l'achat de « douze barillez d'eau rose de Damas [...], comme en une fiole d'or garnie de pierreries, mis au retrait de la reine » (V. GAY, Glossaire archéologique du Moyen Âge et de la Renaissance, revu et complété par Henri Stein, Paris, 1928, t. II, p. 299, entrée « retrait », cité dans N. FAUCHERRE, J. MESQUI, «L'hygiène... », p. 62). 


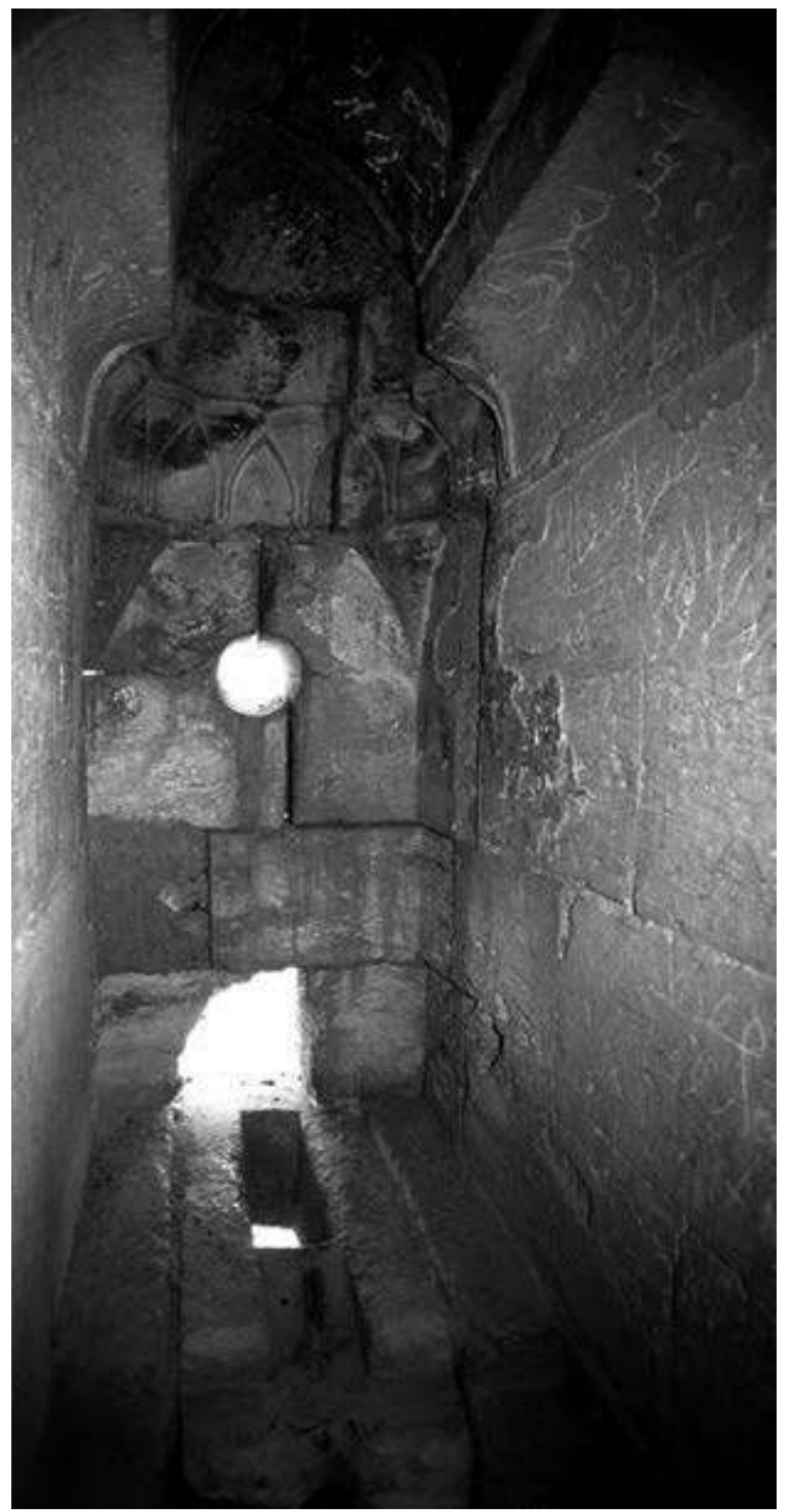

Fig. 13. Shayzar. Latrines ornées de la tour mamelouke (Photographie C. Yovitchitch) 
par les ordres militaires, peuplées de moines-soldats rompus à la vie en collectivité). Les pôles d'hygiène ainsi constitués permettaient de tenir « à l'écart » les bâtiments dédiés aux activités humaines polluantes.

Cyril Yovitchitch - UMR 8167 Islam médiéval

\section{Soulagement au château : les lieux d'aisance dans les fortifications du Proche-Orient médiéval}

Cet article propose une brève immersion dans les latrines des forteresses médiévales proche-orientales à partir d'exemples issus du Bilād al-Shām et de l'Égypte. Dans les forteresses, les lieux d'aisance se devaient de répondre à deux impératifs : remplir la fonction impérieuse de soulagement des militaires, ne pas fragiliser le dispositif défensif pour autant. II fallait qu'elles soient situées dans des endroits rapidement accessibles, aussi bien depuis les lieux de vie que de combat. Néanmoins, cette proximité ne devait pas poser de problèmes d'hygiène ni de pollution visuelle ou olfactive, l'encasernement ne rimant pas nécessairement avec rusticité. D'ailleurs, parfois, les latrines témoignaient du raffinement du cadre de vie du maître des lieux.

Croisé - forteresses - Islam - latrines - Proche-Orient

Relief in the Castle : The Latrines Rooms in the Near Eastern Medieval Fortifications

This paper proposes a short immersion in the latrines of the Near-Eastern medieval fortresses based on examples taken in the Bilād al-Shām and Egypt. In the fortresses, toilets had to meet the dual imperative to fulfil the compelling function of the relief of the soldiers without weakening the defensive system. They had to be situated in places quickly accessible, as well as from places of living and fighting. Nevertheless, this proximity was not to pose problems of hygiene, nor visual or olfactive pollution, as living in military barracks does not necessary go with rusticity. Moreover, sometimes, the latrines testify the refinement of the living conditions of the master of the place.

Crusader - Fortresses - Islam - Latrines - Near-East 
